\title{
FIELD QUALITY IN THE TWIN APERTURE D2 DIPOLES FOR LHC UNDER ASYMMETRIC EXCITATION*,
}

\author{
$\underline{\text { A. Jain }}{ }^{\#}$, P. Wanderer and E. Willen, BNL, Upton, NY 11973-5000
}

\section{Abstract}

Twin aperture D2 magnets are one of the several types of dipoles to be built by BNL for the interaction regions of LHC. To minimize the number of dipole correctors required in the interaction regions, D2 will also be used as part of the steering system. Consequently, the operating fields in the two apertures may differ by up to $\sim 10 \%$ at $7 \mathrm{TeV}$ operation and $\sim 33 \%$ at injection in order to compensate for the strengths of the correctors that would otherwise be required. Such asymmetric excitation of the two apertures may induce undesirable field harmonics. The saturation behavior of various harmonics is studied using POISSON and OPERA-2D. It is shown that the changes in harmonics resulting from the anticipated asymmetry are within tolerable limits.

\section{INTRODUCTION}

As part of the US-CERN collaboration for the Large Hadron Collider (LHC), Brookhaven National Laboratory (BNL) will build a number of superconducting insertion magnets. There are six different styles of dipoles, all with a magnetic length of $9.45 \mathrm{~m}$. Also, all dipoles will use the same $80 \mathrm{~mm}$ aperture coil design that was used for the RHIC arc dipoles. In the twin aperture D2 and D4 magnets, the dipole field points in the same direction in the two apertures. This presents design challenges, particularly for the D2/D4B magnets where the separation between the two apertures is only $194 \mathrm{~mm}$. Preliminary magnetic designs of all the dipoles to be built by BNL can be found in reference [1].

In order to minimize the number of dipole correctors required in the interaction regions, it was decided to use D2 as part of the steering system also. Consequently, the operating fields in the two apertures may differ by up to $\sim 10 \%$ at $7 \mathrm{TeV}$ operation and $\sim 33 \%$ at injection in order to compensate for the strengths of the correctors that would otherwise be required. Such asymmetric excitation of the two apertures may induce undesirable field harmonics. The saturation behavior of various harmonics under asymmetric excitation is studied in this paper.

\section{MAGNETIC DESIGN OF D2 DIPOLES}

A preliminary design [1] of D2 dipoles minimized saturation induced harmonics, but was based on tentative locations and sizes of bus slots and other cut outs needed in the iron yoke. It was assumed in this optimization that the two apertures will be operated at the same dipole field. Subsequently, the locations of bus slots and various cut

\footnotetext{
* Work supported by the US Department of Energy under contract no. DE-AC02-98CH10886.

\#_jain@bnl.gov
}

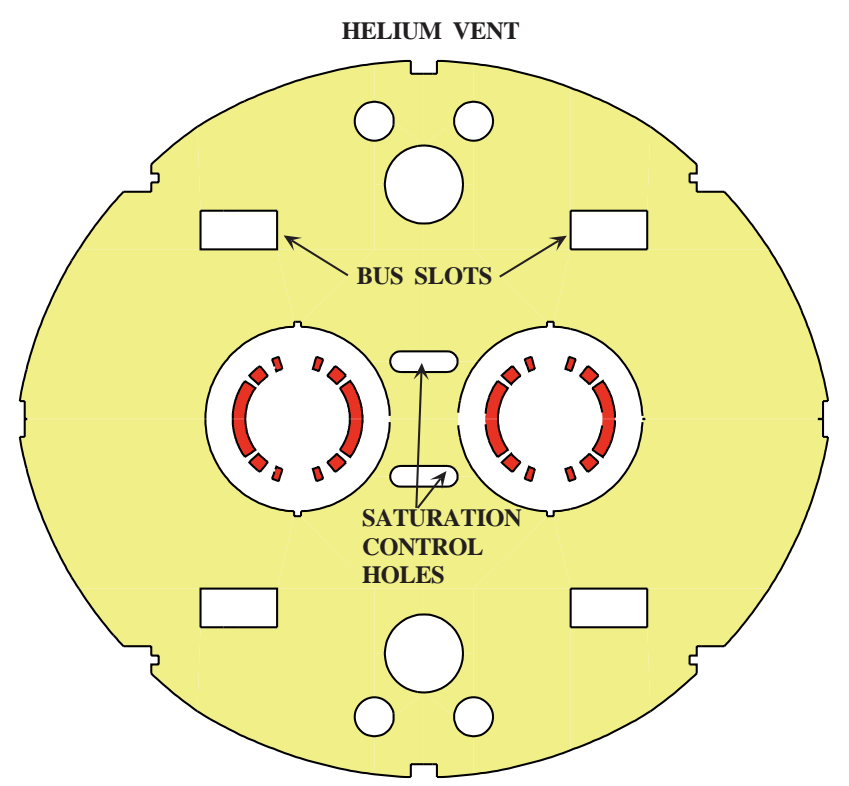

Fig. 1: Design of the D2 cold mass with the relocated bus slots and other cut outs.

outs were changed significantly from the original design due to engineering reasons. This required a new optimization of the yoke for the twin aperture magnets. The current design for the D2/D4B magnets is shown schematically in Fig. 1. In particular, the saturation control holes near the center of the yoke had to be enlarged to compensate for the proximity of the bus slots. Instead of rectangular holes of the original design, holes with semicircular ends were used in order to maintain sufficient distance from the collars. This design was obtained by minimizing current dependence of various harmonics, calculated using POISSON. Only one fourth of the magnet was modeled to obtain maximum precision. This implied equal currents for the coils in the two apertures.

\section{ASYMMETRIC EXCITATION OF D2}

As mentioned before, it is planned to use D2 as part of the steering system in order to minimize the number of dipole correctors required in the interaction regions. The required correction strength is 0.55 T.m at injection and 2.5 T.m at $7 \mathrm{TeV}$ operation. Assuming a magnetic length of $9.45 \mathrm{~m}$, these strengths correspond to $0.0582 \mathrm{~T}$ in the $\mathrm{D} 2$ magnet at injection and $0.265 \mathrm{~T}$ at $7 \mathrm{TeV}$. The lowest operating fields for D2 (at regions IR1 and IR5) are $0.176 \mathrm{~T}$ at injection and $2.74 \mathrm{~T}$ at $7 \mathrm{TeV}$. Thus, the correction strength amounts to $\sim 33 \%$ of the field at injection and $\sim 10 \%$ of the field at $7 \mathrm{TeV}$.

Since the iron in the yoke is far from saturation at the very low fields at injection, even an asymmetry of $33 \%$ 
in the excitation of the two apertures is not expected to produce any undesired harmonics. However, at high fields, the iron is saturated and an asymmetric powering is likely to produce a different current dependence in various harmonics.

In order to study the effect of asymmetric excitation, it is necessary to model at least one half of both the apertures, thus doubling the problem size. This made it difficult to obtain precise results at all values of excitation with the currently implemented problem size limits in the BNL version of POISSON. Consequently, the problem was also analyzed using the program OPERA-2D, which permitted a finer mesh. Good agreement was found between the results of OPERA-2D and POISSON for the cases where the POISSON solution converged.

In order to obtain a conservative estimate, a current asymmetry of $15 \%$ was studied. This is $50 \%$ over the maximum asymmetry expected at $7 \mathrm{TeV}$ operation. Three cases were studied - (a) equal currents in both the apertures, (b) current in the left aperture higher than that in the right aperture by $15 \%$ and (c) current in the left aperture lower than that in the right aperture by $15 \%$. Fig. 2 shows a sample output from OPERA-2D.

\section{RESULTS}

For each of the three cases studied, the harmonics were calculated with the center of the right aperture as the origin. The harmonics, in "units", are defined according to the expansion

$$
B_{y}+i B_{x}=10^{-4} \times B_{0} \sum_{1}^{\infty}\left(b_{n}+i a_{n}\right)\left(\frac{x+i y}{R_{r e f}}\right)^{n-1}
$$

where $B_{0}$ is the strength of the dipole field, $R_{\text {ref }}$ is a suitable reference radius, chosen to be $17 \mathrm{~mm} \mathrm{[2],} \mathrm{and} x$ and $y$ are the horizontal and the vertical displacements from the chosen origin (center of the right aperture). In practice, the normal and skew coefficients, $b_{n}$ and $a_{n}$, were obtained by a Fourier analysis of the $B_{\theta}$ component at a radius of $35 \mathrm{~mm}$, and then scaled to $17 \mathrm{~mm}$. Due to a topbottom symmetry in the model, the skew coefficients were zero.

The low field transfer function is $0.6345 \mathrm{~T} / \mathrm{kA}$ for all the three cases. Fig. 3 shows the change in transfer function (from the low field value) in the right aperture as a function of the dipole field in this aperture. For a given

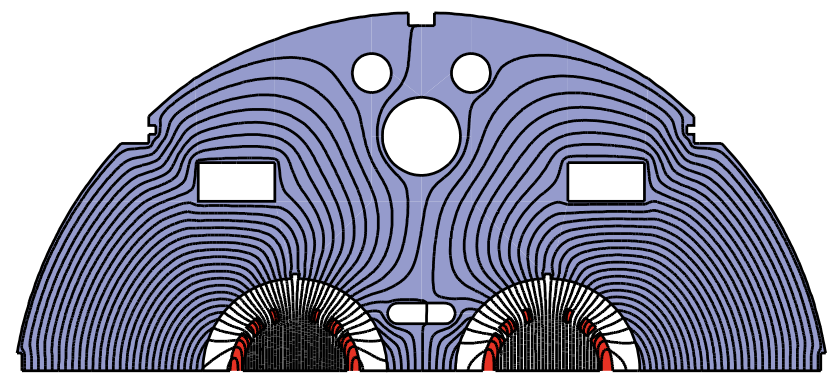

Fig. 2: Field lines calculated using OPERA-2D at $3.77 \mathrm{~T}(6 \mathrm{kA})$ in the right aperture, with $15 \%$ more current in the left aperture

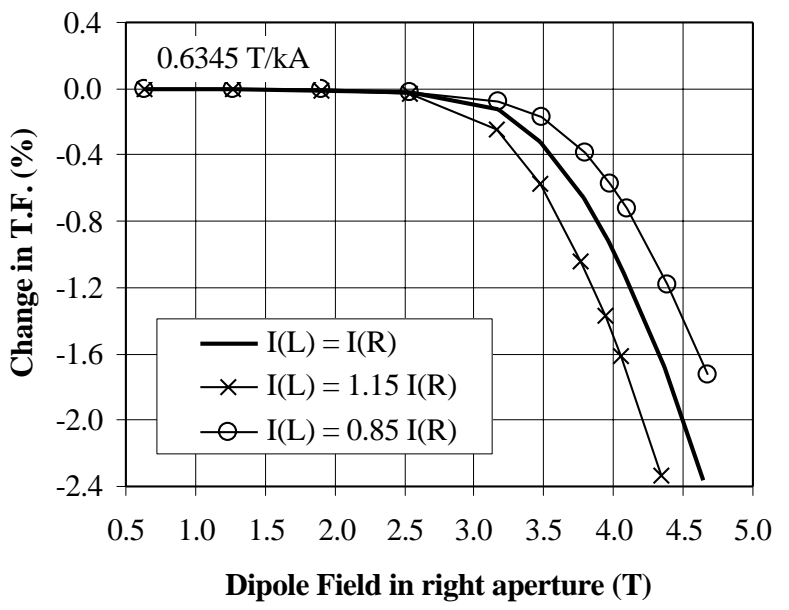

Fig. 3: Changes in transfer function from the low field value as a function of dipole field for various asymmetries in excitation of the two apertures.

dipole field, there is a bigger drop in transfer function when the other aperture is powered with a higher current, as compared to the case of a symmetric powering. A $\pm 15 \%$ asymmetry in excitation gives rise to a $\pm 0.4 \%$ change in transfer function at $\sim 6 \mathrm{kA}$. This implies that the current required to achieve the same field in a given aperture may vary by up to $\pm 23 \mathrm{~A}$ at $3.8 \mathrm{~T}$ depending on the excitation level of the other aperture.

The saturation behaviors of various harmonics ranging from quadrupole to 14-pole are shown in Figs. 4 through 9. For each harmonic, any systematic value at low fields is subtracted out to obtain the contribution from iron saturation alone. As can be seen from these figures, the saturation induced harmonics are well below 1 unit in the case of symmetric excitation (solid lines) up to the maximum operating field of $3.83 \mathrm{~T}$ for these dipoles (7 $\mathrm{TeV}$ operation). In the case of asymmetric excitation, the lowest order quadrupole and sextupole harmonics are the most affected. Even for these harmonics, it is seen from Figs. 4 and 5 that the saturation induced harmonics

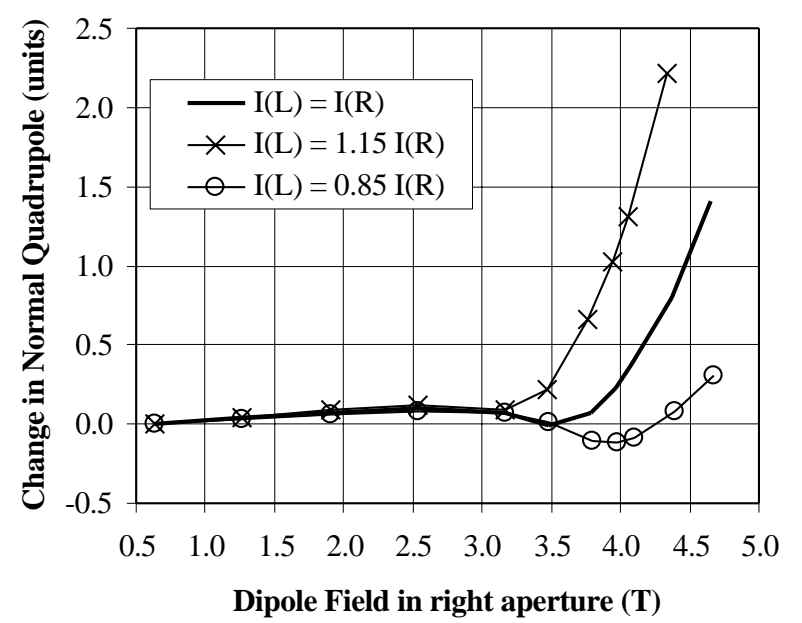

Fig. 4: Saturation behavior of the quadrupole term 


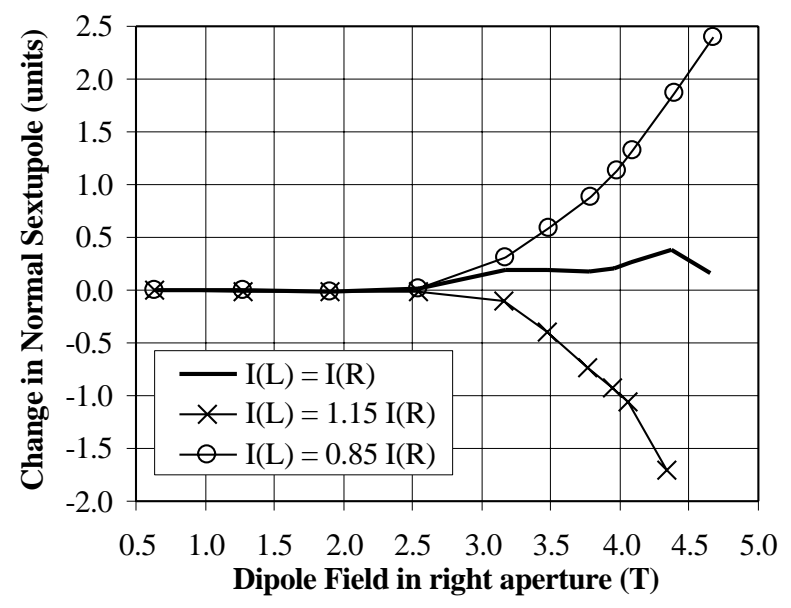

Fig. 5: Saturation behavior of the sextupole term

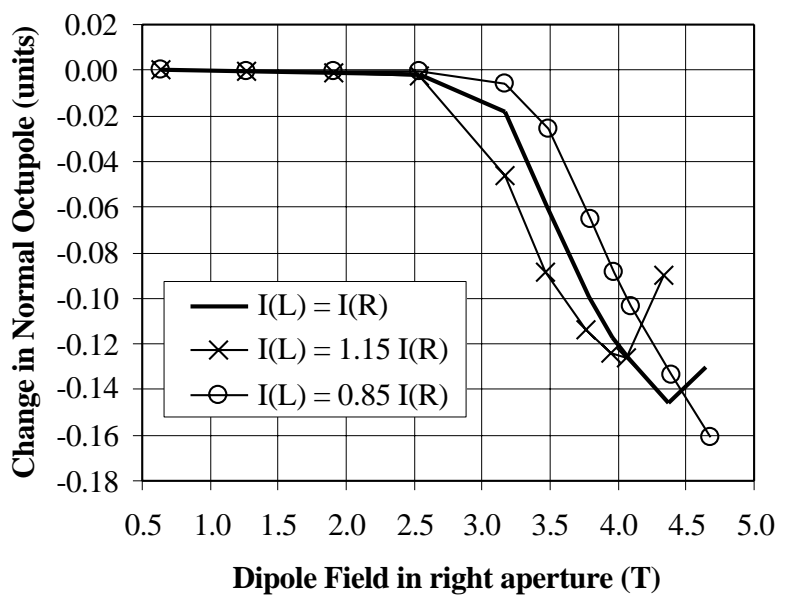

Fig. 6: Saturation behavior of the octupole term

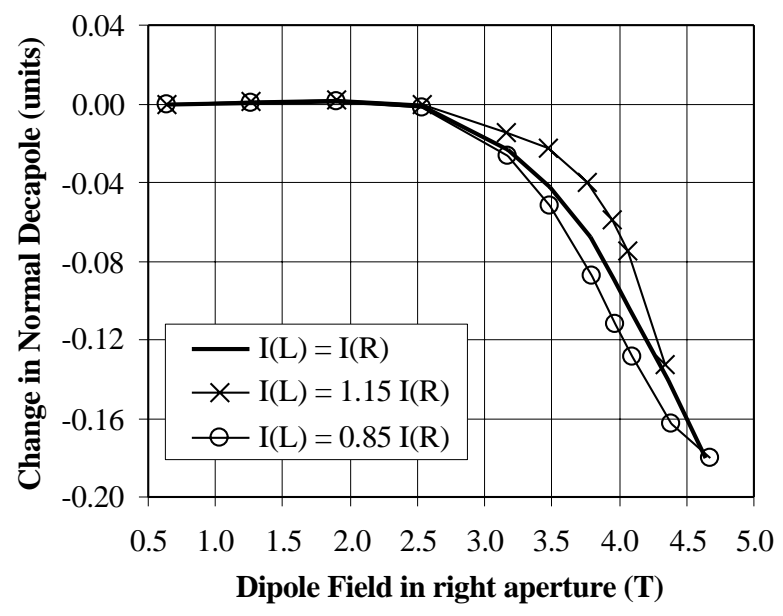

Fig. 7: Saturation behavior of the decapole term

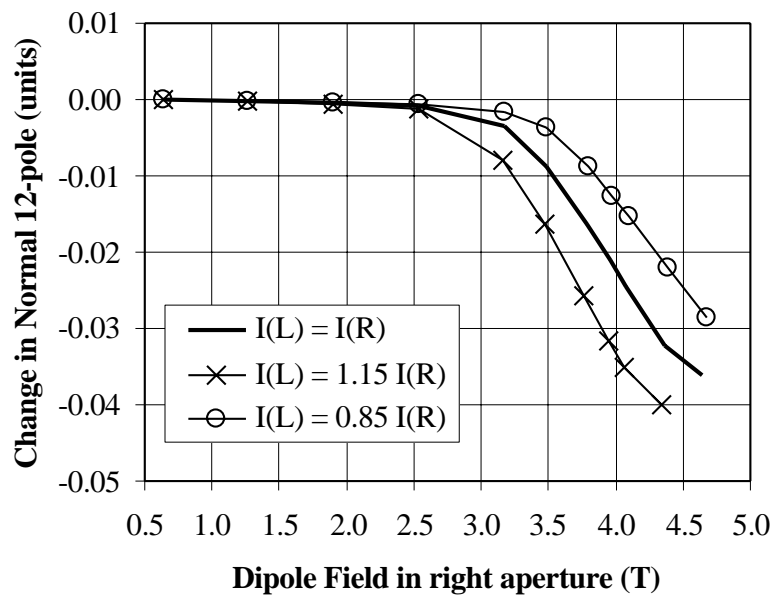

Fig. 8: Saturation behavior of the 12-pole term

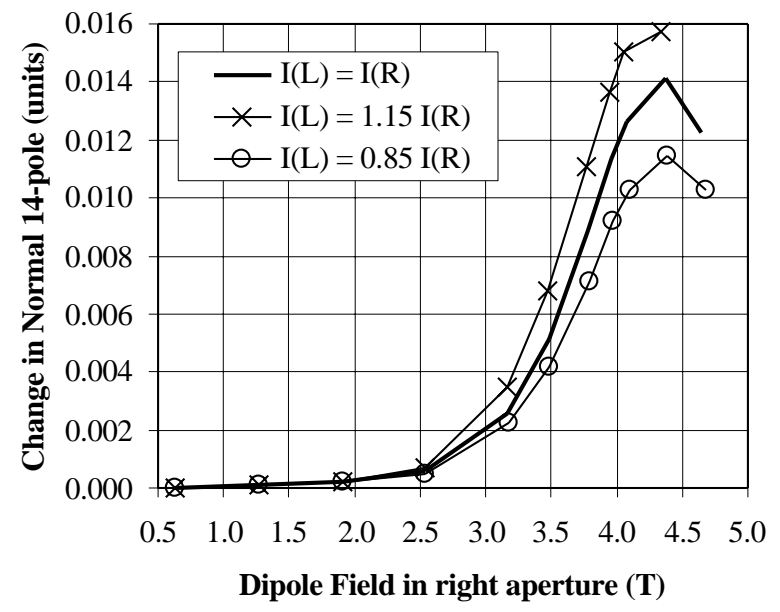

Fig. 9: Saturation behavior of the 14-pole term

are still below 1 unit at the highest operating point. Since the actual asymmetry needed in operation is expected to be less than $10 \%$, and that too at a lower field, the small deterioration in the saturation behaviour should be quite acceptable. For the higher order terms, octupole through the 14-pole, the changes are at the level of 0.02 unit or less. These changes also are not expected to limit the performance of the $\mathrm{D} 2$ dipoles.

\section{ACKNOWLEDGEMENTS}

We thank S. Kahn and B. Parker for their help with the OPERA-2D calculations.

\section{REFERENCES}

[1] A. Jain, et al., "Magnetic Design of Dipoles for LHC Insertion Regions", Proc. EPAC98, pp. 1993-5.

[2] R. Wolf, "Field Error Naming Conventions for LHC Magnets", CERN report LHC-MMS/ES/01 